\title{
Image Dissection in Natural Scientific Inquiry
}

\author{
Karin Knorr-Cetina \\ Klaus Amann \\ University of Bielefeld
}

\begin{abstract}
Images are objects of work in the laboratory. On its face, this work is achieved through talk Yet the talk attached to these images makes reference to other inages, which are drawn from various environments. In this article, four such environments are identified: the domain of laboratory practice; the context of invisible physical reactions; the future inage as it will appear in publication; and the domain of case precedents and reference scenarios from the field. The work of image analysis brings the outside of the image into it and takes the inside out. Thus it can be seen that images are not just taken, they are designed and made.
\end{abstract}

Philosophers, historians, and sociologists of science have long considered writing to be a central part of scientific activities. From an outside observer's perspective on science, this conception of scientific writing is plausible enough. Written articles are the ostensible goal and the raison d'etre of much scientific activity. They are the topic of scientific arguments, the materials that integrate scientific communities, and the carriers of scientific information and "progress" in a field. They are the tangible result of transient and undocumented research activities, the palpable "traces" left for analysts of science to study. Scientific inquiry, from this perspective, is centrally concerned with producing and qualifying texts. ${ }^{1}$ Yet from within scientific inquiry, the focus of many laboratory activities is not texts, but images and displays.

The difference is crucial. Images and texts are differently occasioned and differently handled in the process of inquiry. On occasion, images and texts are assembled together to create one another's referents - in the process of writing up data for the purpose of publication, for example. Yet the very necessity of their assemblage, at the stage of publication, points to their singular relevance in other contexts, such as in inquiry. In a sense, natural

AUTHOR'S NOTE: The research for this article has been made possible by a grant from the Deutsche Forschungsgemeinschaft for the study of "Complex Knowledge Processes." We are grateful to the Center for Science and Technology Studies, University of Bielefeld, for facilitating this research. 
scientific inquiry does not recognize texts as a concrete occupation in experimental processing, though texts may be searched and consulted when experiments are designed or when problems develop, and some verbal passages are inserted in laboratory protocols. ${ }^{2}$ Natural science laboratories are not text-processing laboratories. On the other hand, many natural scientific areas appear to be centrally occupied with images and displays. We consider these to be figurative representations, in contrast to verbal representations.

This article is about the role of images as a topic and a resource in laboratory practice. ${ }^{3}$ Our goal is to describe the work performed on and with images in natural scientific inquiry, as exemplified by autoradiograph analyses that are routinely and typically performed in molecular genetics labs. Autoradiographs are generated by radioactively labelled DNA or RNA fragments separated in an electrophoresis gel on which an X-ray film has been exposed. Autoradiographs have many purposes. In the study of transcriptional control mechanisms, they are employed to determine the effect of DNA regulatory elements that "enhance" or "promote" transcriptional activity during the transcription of DNA into RNA and that are, for that reason, relevant to the understanding of normal and abnormal cell growth. Autoradiographs display the length of the RNA that results from a presumed enhancer or promoter effect through the position and size of dark "bands" (spots) in the relevant "lanes" (columns) of a transparency-like film (see Figure 1). Typical experiments, in recent years, have attempted to isolate segments of viral DNA that are responsible for a proposed enhancer or promoter effect.

The data presented in this article have been derived from an ongoing study of molecular genetics conducted since September 1984 at the Center for Molecular Genetics in Heidelberg and at the Max Planck Institute for Bio-Physical Chemistry in Goettingen, FRG. ${ }^{4}$ The group studied works on transcriptional control mechanisms; in this field, it is one of the leading research units in the world. Most of the examples presented below were drawn from a series of interconnected experiments involving a particular method of RNA preparation ("S1 analysis").

\section{The Transparency of Images}

Autoradiographs of the type discussed above furnish marked occasions for image work. They are "noticeable"; their occurrence in the lab attracts the attention of participants, who join the author of the autoradiograph in inspecting the film against the light. Autoradiographs represent experimental 


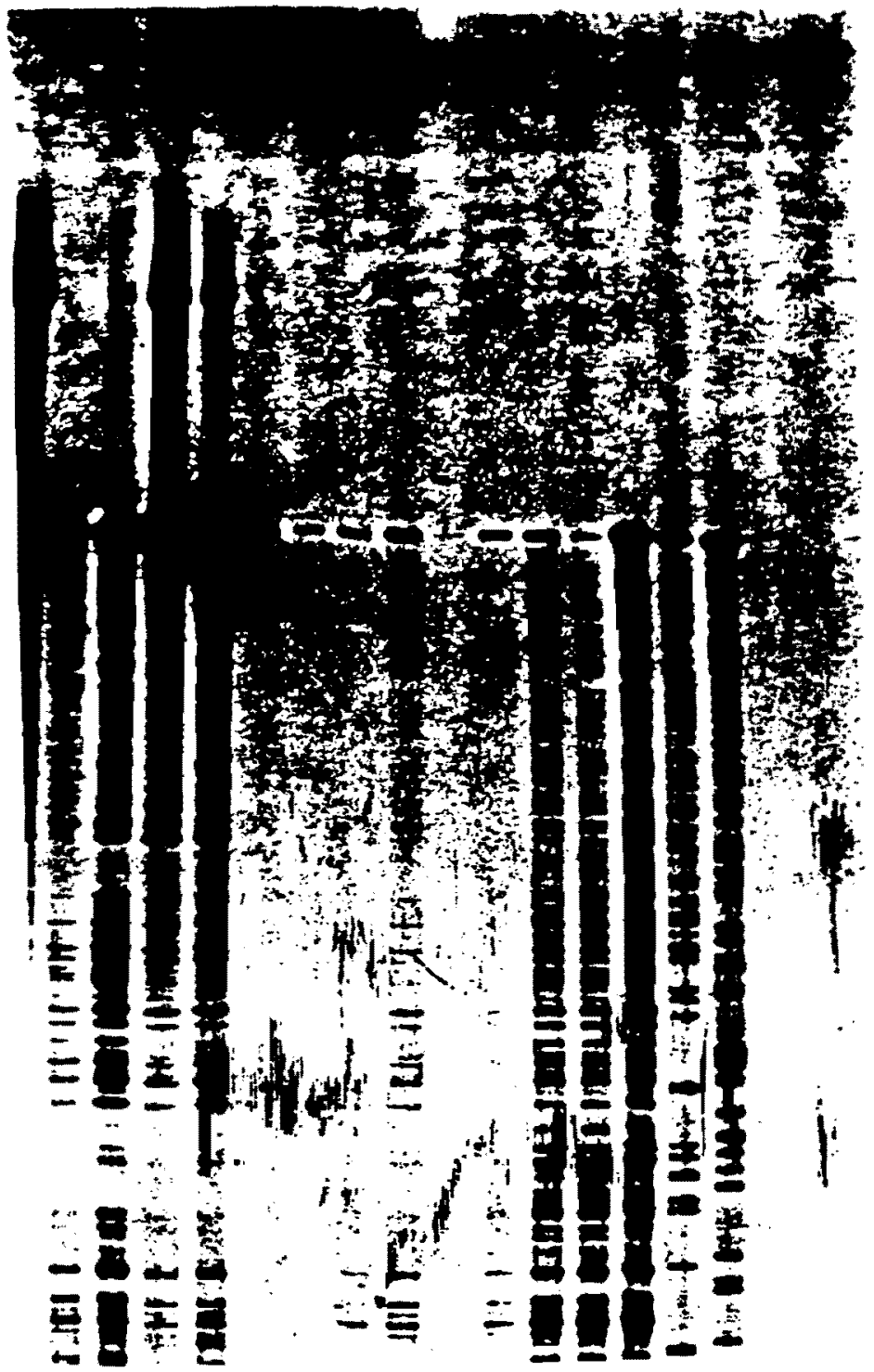

Figure 1. Example of an autoradiograph. 
outcomes or "data." Their attraction derives from the fact that they indicate whether a correct "start"6 of a process of transcription has occurred and how much RNA was transcribed. It is tempting to associate such images with closure - with the closure of research activities and of experimental questions, of scientific arguments, and of processes of persuasion. Much like the articles in which they are published, these images can be seen as endpoints of inquiry processes, as data "displays" that provide answers, rather than pose questions, or as "pictures" of experimental effects that play a definitive role in settling doubts about knowledge claims. And, indeed, scientists treat such images as answers. For example, they treat the above autoradiographs as reference scenarios for how experiments can "go" and as points of departure for future work. But they do not treat them as closed objects. More precisely, they treat images as self-contained subjects that carry the analysis of an event within them. Images are objects on which work is performed in the laboratory; like other materials handled in the stream of laboratory activities, they are processed. The analysis contained in a data display is not written on the image's face. It is brought to the fore by means of image analysis techniques that look behind the surface of the features displayed. Participants look at the display as one would look through a window that opens to a whole new environment of processes and events.

Consider an analogy. When mouse number 385 - the first transgenic mouse obtained in the laboratory studied, in which a mutation had been induced through the microinjection and integration of foreign DNA - died during the night of 5 November 1987, scientists decided to perform an autopsy. They tumed the mouse on its back, spread-eagled the animal by fastening its paws on a piece of cardboard, opened the skin with a pair of scissors, "unclothed" the inside by pulling back the fur with surgical forceps, and proceeded to dissect several organs. The point of pathology work was to penetrate beyond the surface of the animal to identify directly the effects that transgenity had produced on the organs, the spine, and other locales of cell differentiation. In other words, the purpose was to look directly at the intemal and normally invisible processes triggered by the mouse's transmuted gene. Like the processes of regulation and deregulation of cell differentiation, most objects and processes of interest to scientists are "unreal" in the sense that they cannot be observed or analyzed in real time, that is, while the processes or events are still active.

Like the dead mouse, data displays - such as autoradiographs - are objects on a dissecting table on which the autopsy of the events of interest to scientists is performed, once these events cease to be alive. The display 
carries on its surface the signature of these events; it is the result of an imaging technology that creates visible traces of invisible reactions. Yet, as argued elsewhere,' with original data these signatures tend to be unclear. The marks on a film or on photographic paper are initially signs without meanings or referents; these signs must first be attached to the objects that they are presumed to represent. This process of assembling a visual trace with a suitable referent proceeds through image dissection. It involves pursuing the threads that lead from bits and pieces of the surface of the display to developments and occurrences underneath.

What dissecting instruments do scientists employ in this process? On the face of it, the answer is "talk." Scientists who join each other in inspecting an autoradiograph against the light invariably begin to talk. Below, we will provide several examples of such talk. Elsewhere, we have described the formal conversational routines scientists employ in "fixing" the evidence on a data display, that is, in associating visual signs with the objects these signs are going to represent. ${ }^{\mathrm{B}}$

Yet this analysis does not consider the tools in terms of which talk itself achieves its work. As it appears, the talk that performs image analysis, in turn, makes references to images; it invokes pictures, images, and "impressions," a repertoire of chunks of visual and experiential knowledge not adequately describable in terms of verbal representations. Image inspection, as we encounter it in the laboratory, appears to involve a conversation of images, not just of words. By and large, each verbal exchange attached to a technical image can be fully translated into a visual/experiential script. It is a script made up of congeries of visual and experiential meanings, of scenes in mind and pictures on file, that are derived from laboratory activities, disciplinary exchange, and education. How do we know about the existence of this script? Images are frequently invoked in verbal exchanges through direct reference, for example, through a reference to somebody else's autoradiograph or to a phenomenon observed in the lab. They are also invoked through the visual and experiential definitions of technical notions, and through scenic descriptions (see later sections). Participants dispose of a whole repertoire of images and scenic experiences that "spell" their verbal interaction. These images account for the curious "gestural" quality of participants' talk. Scientists rarely argue in image-attached conversations. In dissecting the object, they prefer to point. ${ }^{9}$ They point to images and experiences that are communally invokable and sometimes jointly constructed symbols of processes and events. These are the visual and experiential props through which a communal train of thought, clothed in words, proceeds. 


\section{The "Insides" of the Image}

Let us be more precise. What is it that participants see when they look through an autoradiograph display, that is, when they invoke the above mentioned visual and experiential props? Displays treated as transparent in image analysis bring to view not just one but several environments. These are reference contexts routinely and systematically lifted to the fore through the dissecting instrument of talk. Four such domains can be identified; a fifth must be added if we consider that the image, at times, provides its own context of analysis (see Figure 2):

1. The domain of embodied laboratory processes, from which the image has arisen and which is recalled in terms of a vocabulary of scenic descriptions and visual/experiential definitions.

2. The domain of invisible experimental reactions and events formulated through formal glosses and a visual language of technical design.

3. The domain of publication, in regard to which the proto-data of the laboratory are transformed into evidence exhibits.

4. The domain of reference scenarios and case precedents on file or "in the mind," which are invoked for the purpose of guiding or firming up laboratory strategies.

5. The domain of the image, scrutinized whenever it provides its own environment for inspection.

The above domains are invoked in terms of the images and "scenic" notions that make up the above mentioned visual/experiential script. They are looked at, rather than talked about, in abstract terms. More concretely, features of the autoradiograph display are attached to details of the above reference contexts as envisaged or experienced by participants involved. Each of the first four domains has its own set of preferred images and experiences in terms of which it is called forth. The fifth, the image treated as transparent in regard to these domains, can also be looked at as a context of its own.

\section{Revisiting the Lab}

Consider the domain of embodied laboratory practice. The laboratory is invoked when scientists consult their memory of a procedure in order to identify and evaluate visual traces on the display. Reference to the lab is made in image-analysis talk through experiential definitions and scenic descriptions. A substantial portion of the technical vocabulary used by participants appears to have experiential meanings, in the sense that it is learned by doing 


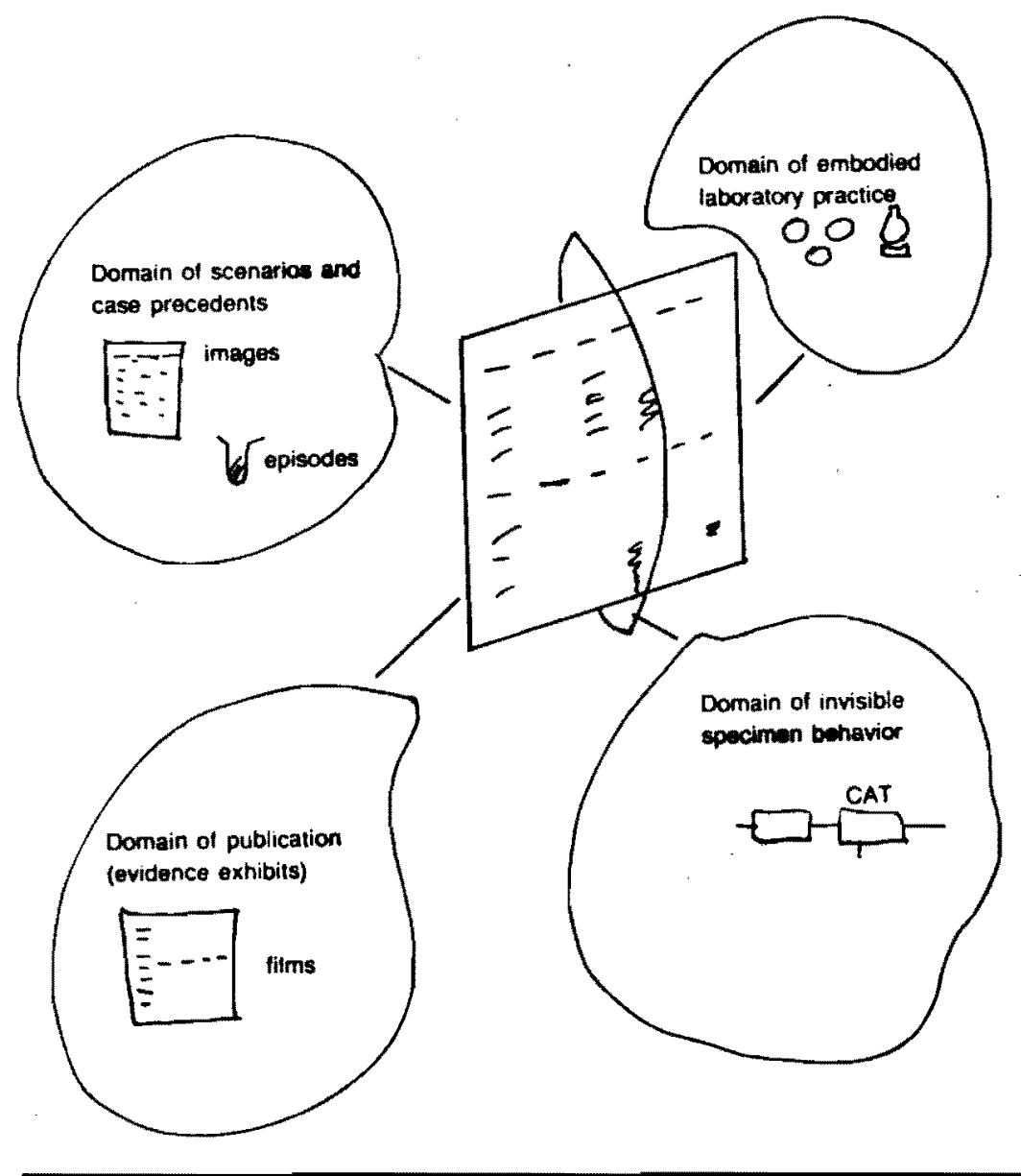

Figure 2. Looking through an autoradiograph.

or by being shown how to proceed. Accordingly, many technical notions refer to specific sequences of embodied practices, for example, to the kind of thing one does when materials must be "centrifuged through a Caesium-chloride cushion," when one "pours" or "loads" a gel, or when one "shoves twice as much S1" into a test tube. Centrifuging through a Caesium-chloride cushion, for example, means filling a test tube with Caesium-chloride before centrifuging, to create a medium within which RNA, DNA, and a pellet can separate and form distinguishable bands. Experiential definitions may not be 
visual, but neither are they verbal. Moreover, visual impressions that pick out significant aspects of embodied practices are always close to hand. For example, in references to a gel run, the image of a gel as an observable entity in the laboratory (e.g., $21 / 2 \mathrm{ft}$. by $1 \mathrm{ft}$. glass plates attached parallel to each other with the transparent polyacrylamid gel inserted between them) may become visually relevant, as in the following utterance, in which the second speaker relates what he saw in the lab to what he sees on the autoradiograph film in his hand. ${ }^{10}$

$10015-2$

32 Jo ((points to bands on the film)) ok, if this is 600 ... and this 400 , then 300 would be here. ((Ea shakes his head?)) no

34 Ea Well no, the gel ran so that blue was here ((points to film)), and this is 130 .

In inspecting the film, the author recalls his visual impression of where on the gel in the lab the blue marker (a blue horizontal band running across the gel) appeared when he stopped the gel run, a position he points out on the film and identifies to be "130." Such references to observable entities and occurrences in the lab can be called scenic descriptions. In contrast to the technical notions mentioned above, they circumscribe technical occurrences in terms of a vocabulary that preserves the visual/experiential quality of embodied laboratory practices. Scenic descriptions specify and detail jointly invoked experiential meanings. In the following exchange, Ea specifies the procedure used in determining the amount of RNA applied in a gel run in terms of a visually/experientially salient vocabulary.

$10015-2$

65 Jo why?/didn't you determine the UD ((amount of GTC RNA))?

66 Ea naw, I know exactly how much it was

67 Jo ((inaudible))

68 Ea this is from four dishes, this is from two dishes. My/now

I am doing the spinners, $25 \mathrm{ug}$... not much.

Ea refers to the number of cell culture dishes from which he collected the cells used in making a RNA extraction. In other words, he refers to what is pictured in Figure 3.

Ea indicates concrete objects in the laboratory, not a numerical quantity. These are the dishes in which cells transfected with plasmids are grown until a sufficient amount of RNA has been transcribed. The vocabulary he uses is visually and experientially descriptive, rather than technically precise. Scenic descriptors may be borrowed from everyday life, such as when partici- 

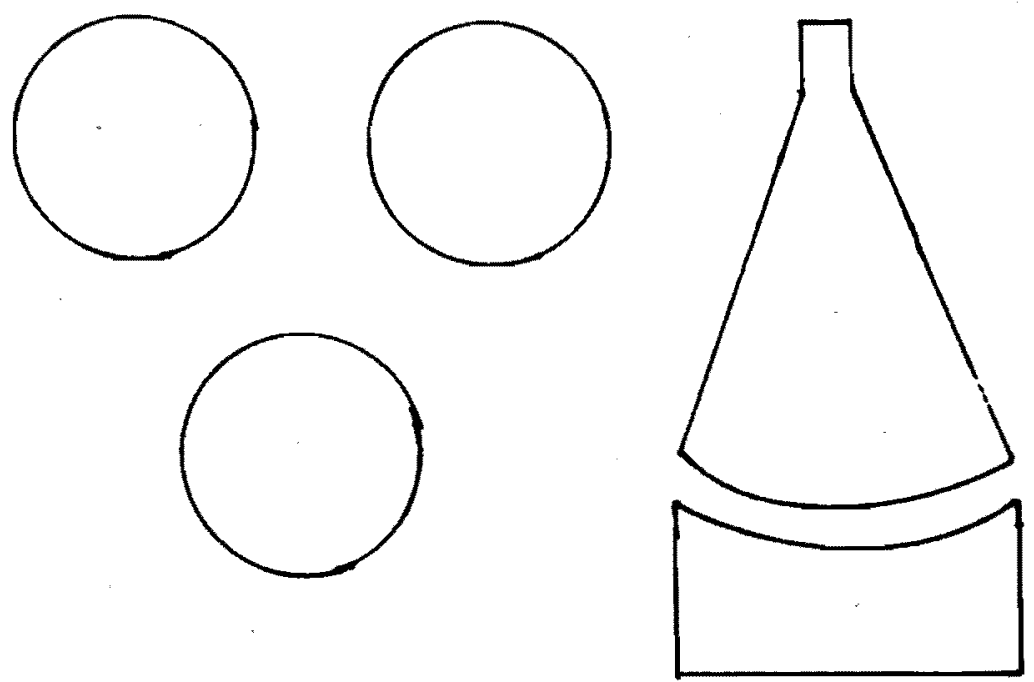

Figure 3. Cell culture dishes and spinner.

pants refer to the bands on an autoradiograph film as "fat," "tiny," "strong," or "weak." Narrative scenic descriptions are sometimes found in laboratory protocols where they depict sensory impressions participants record when they inspect problem occurrences.

It is important to note that visual/experiential clues are built into experimental procedures as signals to be noticed by the researcher. They are there to be consulted and to be recalled if the need arises. The blue marker mentioned before was a purposefully built-in visual signal. No less important are the signals that are given off by experimental processes without being deliberately included and, for the most part, without being clearly spelled out in procedural descriptions. An experimental scientist must leam to acquire a good sense of what these signals are and how they ought to be read. It is an embodied sense that produces a virtual knowledge: Many of the hints that a trained and observant participant picks up during an experiment are not made explicit by laboratory members. They surface in the above mentioned visual/experiential script, but this script is assembled only when participants inquire into (search their memory of) the performance of a procedure. The capacity to pick up visual/experiential information is considered crucial by participants; for example, they insist that doing an experiment oneself is necessary to be able to produce valid judgments on its outcomes." 


\section{Glossing the Invisible}

Scientists look through an autoradiograph into the laboratory when they analyze the technical image; they recall the visible real-time experimental operations and the "sense" they acquired of the performance of a procedure. But they also look through the autoradiograph into the realm of the invisible experimental processes and occurrences whose signature they find on film. A second domain that participants call for and scrutinize in image dissection is the domain of these processes. These are biochemical reactions in test tubes, laboratory dishes, and similar utensils envisaged and formulated through a language of design. The images invoked in this connection are formal glosses; they consist of construction diagrams, design maps, or conceptual reconstructions of processes and events. Accordingly, the issues relevant in this context can be seen to be related to the architecture of a phenomenon or process, for example, to the question of where in a plasmid a start must have occurred for the band on the autoradiograph to show a certain length. A typical formal gloss or "design" of an occurrence is shown in Figure 4, which, for participants, defines what is meant by a "start in CAT."

A "start in CAT" refers to an RNA transcript that has been initiated within the CAT gene, rather than between the CAT gene and the regulatory elements, in a plasmid into which these items have been inserted to produce and measure transcriptional control effects. For all practical purposes, a "start in CAT" is defined by the illustration presented above. No scientist will ever attempt to explain what he or she means by a "start in CAT" without resorting, if possible, to a more or less elaborate version of this drawing, or at least to a gestural rendering thereof. Just as a substantial portion of the technical vocabulary that participants employ has experiential definitions, so an equally substantial portion is visually defined through design maps such as Figure 4. The meaning of many technical notions is learned through figurative representations in textbooks, on blackboards, and on scraps of paper where they are jotted down during explanations. It is these visual formulations that are referenced in problem discussions and that are expanded into a figurative language of technical design suitable to envisage, or to visually "talk about" invisible processes and events. When they work out the meaning of signs on an image like an autoradiograph, participants employ this language as a shorthand everyone understands (see Figure 5).

\section{Looking at the Future: The Context of Public Appearance}

The third domain looked at through the window of an autoradiograph display is the domain of publication. In dissecting the image, participants 


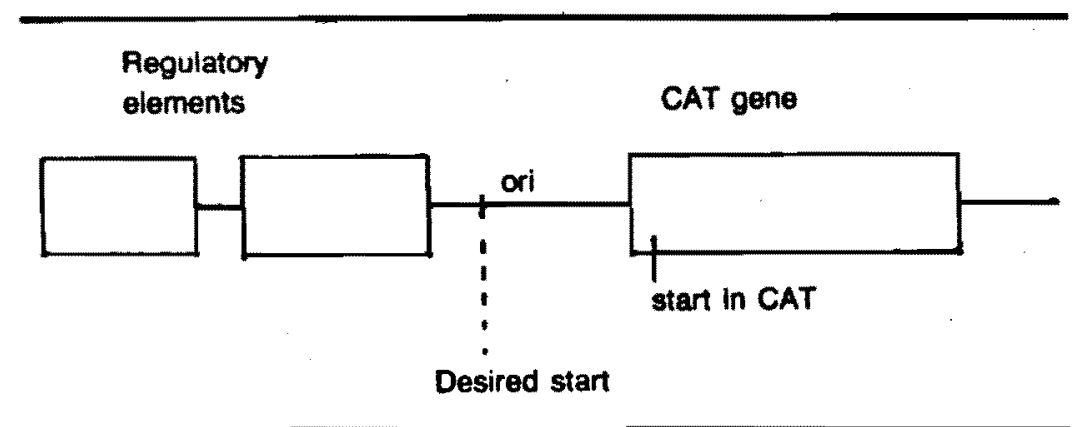

Figure 4. Viaual definition of "start in CAT."

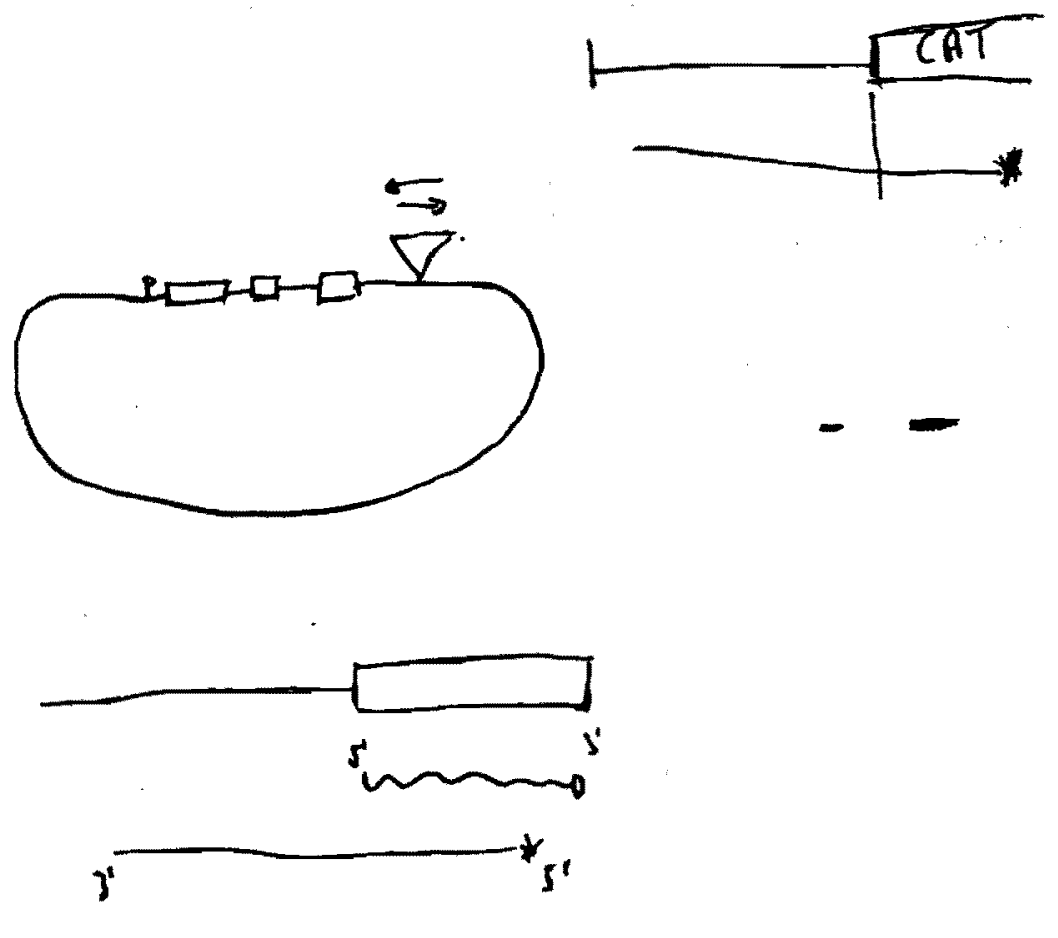

Figure 5. Genetic "design" language on scraps of paper used by participants during image analysis. 
repeatedly turn away from its past and from the "unreal" (invisible) events at its heart to consider the future of the image. How will it have to be trimmed and made up before it can be presented in a paper? There is usually no question of simply leaving the display as it is. Rather, the questions asked relate to the issue of whether and how the image can be made to "shape-up" for purposes of publication. The image is not more than a point of departure for this shape-up. It is a source material that must be developed and enhanced before it can be publicly shown.

The utterances and images that invoke the domain of publication relate to this development; they refer to practices that transform the images scrutinized into evidence exhibits or showable "proofs." When they invoke these practices, participants seem to envisage both the picture as it should look when published and the operations of arriving at this picture, as defined by experiential definitions. Publicly "showable" evidence exhibits require preparatory manipulations that range from photographic touch-ups to repeats of the experiment. To a degree, these practices vary with the kind of image that is to be published. Yet, for any given display, the set of manipulations routinely invoked by participants to arrive at an evidence exhibit are limited and can be clearly specified. In the next section, we give an example of these manipulations.

\section{Scenarios and Pictures}

If a frequent glance at the future an image has in publication characterizes image inspection, so does an occasional glance sideways at other scientists, both inside and outside the laboratory. What is it that they have got, or have done, in a similar situation? Do their visuals or their displays support or contradict the present strategy or interpretation? In working through an autoradiograph, participants occasionally arrive at the provinces of work of others as well as at their own earlier research preoccupations. These works serve as case precedents and reference scenarios in regard to the image that is scrutinized. They are looked at in regard to the precedent they provide for the conclusions to be drawn on the autoradiograph in hand.

Reference scenarios can have a physical reality; they may, for example, be autoradiographs produced in earlier experiments that are somewhere on file in the lab. Or they may be pictures someone has seen and calls to mind in an image analysis discussion. In both cases, the pictures are invoked by direct reference; they are not implied as the images and experiences underlying technical notions that have visual/experiential definitions. Consider the initial exchange in the transcript presented above: 
$1001515 \mathrm{ff}$

58 Ea (...) but the funny thing is, the thing that's really funny is, Jo never sees the probe up there ((points to film))

60 An sure he does

61 Ea he tells a different story

62 An c'mon, he's talking ((inaudible). I saw it with my own eyes on his gels

The passage refers to Jo's film as envisaged by Ea, seen by An, and three days later actually shown around by Jo in a continuing discussion:

$1401505 \mathrm{ff}$

11 Jo this/ I've got this/ I can show you. l've got something similar ((holds up and shows his film))

Some scenarios are "hearsay" examples: One has heard that another member of the lab or a scientist one knows has had certain experiences or has obtained results that one envisages to have a certain relevance. Interestingly, such case precedents are frequently embedded in little stories or story fragments. They are encapsulated in tales of "what I once got when I . . .," "what $\mathrm{X}$ did ...," or "what $\mathrm{Y}$ found ...." At times, the stories are dissolved in question/answer series ("Hasn't $Z$ once done this? ... ," "And then what happened? ...," etc.). Experiences acquire the status of scenarios for future work through being encapsulated in stories that cinculate. The immediate purpose of drawing a case precedent in image analysis is to compare features of the image (or of its architecture and procedural history) with similar materials. On a more general level, scenarios provide a resource: a similar problem, an already worked-out interpretation, or a possible solution arrived at in an analogous case. In this wider sense, many kinds of images and experiences can serve as scenarios. As case precedents, scenarios are potentially relevant to all domains - they may be recalled when participants revisit the laboratory, envisage invisible experimental reactions, or plan the future as they consider publication of an image. In a sense, scenarios do not constitute a reference context independent of others. They make up a repertoire of experiences that are called upon in a way that is mediated by the needs of other domains. For example, scenarios of comparable experiences are frequently invoked for the purpose of substantiating and detailing the strategies adopted in other domains. To illustrate, in image analysis that tums to issues of publication, scenarios (in the form of precedent data displays in the literature) tend to play the role of a firming device. Participants recall someone else's data-exhibiting strategies, as exemplified in his or her pic- 
tures, to substantiate and confirm the editing policy proposed in regard to the publication of their own display.

\section{Working Through the Image: Three Examples}

Image dissection re-creates participants' practice; it displays this practice as a stream of activities that have a history and a purpose, that rely on a case law, and that distinguish between the surface of events and their invisible structure. Through the dissecting instrument of talk, an autoradiograph's environments of past laboratory happenings and future (publication) projects, of disciplinary precedents and underlying biochemical designs are lifted to the fore. Why are they looked at? And what happens when, on occasion, participants stick to the surface of the image and let it provide its own reference environment for the features displayed by the image?

Some answers to these questions have already been given or implied. In the following, we will expand these answers by focussing on three examples of image work. These illustrate the use to which the domains introduced in the last paragraph, the environments looked at in image inspection, are put. To recall, the work achieved through image dissection in the lab is part of the process of fixing the evidence, that is, of attaching referents to the signs on a data display. The details of this work depend on the domain to which it pays attention. All domains appear to be equally available in image inspection in that participants may take a leap into any one of these contexts at seemingly any point in their image-attached conversations. There are, nonetheless, preferred locations for some of the domains to become relevant, and the leap into a particular environment may mark a specific stage in image work.

\section{Image Arithmetic}

Consider the case in which participants pay close attention to the surface of the image without shifting their gaze to the processes or occurrences underneath - that is, without treating the image as transparent. This situation frequently occurs in the beginning of image inspection. Participants tend to approach the image like a tablet of signs that carries meaning on its face. They are forced off this surface and moved to do autopsy work by the "pathologies" they encounter: For example, by bands that are missing on autoradiographs, occur in the wrong places, smear into each other, or have otherwise unintelligible features. While they attend to the surface features of the image, participants formulate and evaluate what they see. 


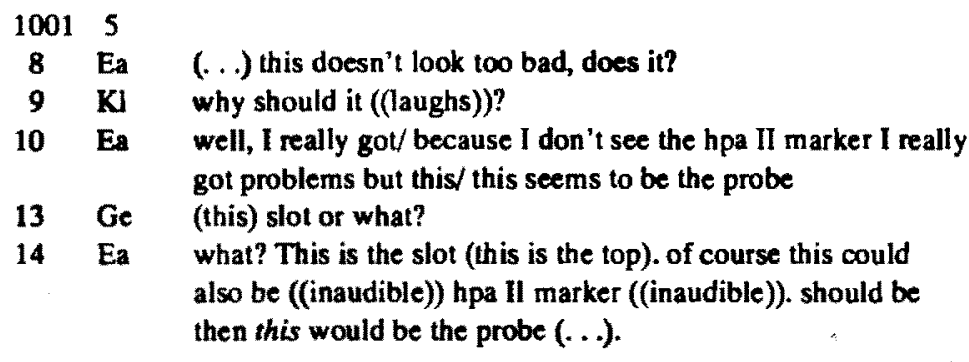

But they also survey the surface; they calculate the length and position of its features by taking known roadmarks as a fixed point, by applying measuring sticks ("markers"), and by resorting to external reference information. For example, somewhat later in the above conversation, Ea identifies the slots on the film in which different genetic constructs have been inserted, while at the same time considering the bands that have appeared in the respective lanes. What follows is an attempt to deduce which band could be the "original start" by taking a presumed marker band, whose length is estimated to be 600 , as a fixed point. The "marker" is a known construct inserted into one lane, from which the length of other bands on the film can be calculated if the marker bands are visible and their length has been determined. ${ }^{12}$ As their gaze climbs down various bands and moves back and forth between these and the presumed marker band, participants perform a visual ground feature computation.

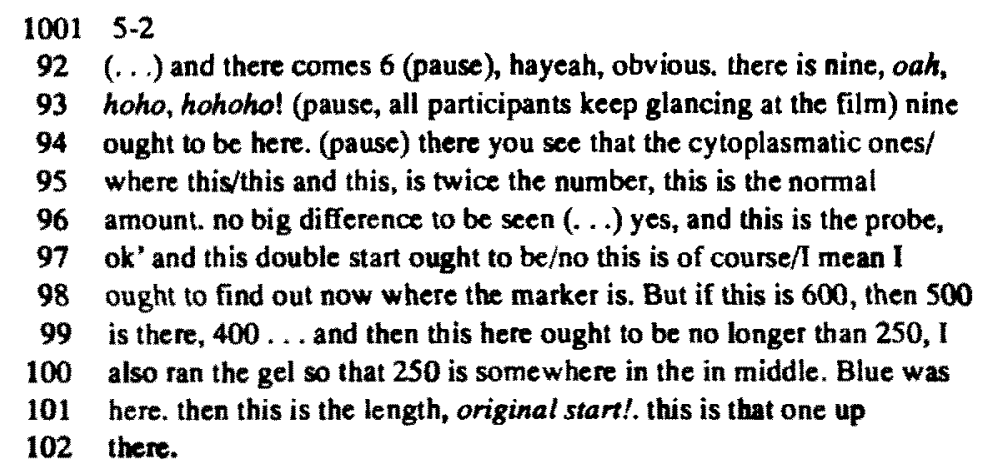

The features of technical images not only pose problems, they also suggest solutions. Image arithmetic is a way to arrive at solutions by exploiting the calculable properties of visual marks on a two-dimensional plane, such as the distance and relative position of these marks, and their measurable height 
and length. The method can be seen as an alternative to the reconstruction of specimen behavior in the lab through which participants also derive inferences about image features - a method we will exemplify next. In the above case, participants arrived at a candidate formulation about the identity of the original start on the film by a combination of basic arithmetic (addition and subtraction) with a stepwise procedure of taking the calculations made for one band as a starting point for the calculation of another.

In later conversations, this candidate formulation will be tested against others by recalling the occurrences in the lab. But, even in image arithmetic, external reference points are brought into the picture at some point to establish and confirm the calculation. In the above example, the author refers to visual features built into the experiment such as the blue marker or his attempted positioning of a band length of 250 in the middle of the film.

Note that image calculations are measurements by eye, done, for the most part, without pencil and paper. What is interesting about this eye work is that the landscape of the image determines the path of the inspection. It suggests certain connections, puts up obstacles, makes it difficult or easy to follow a particular trail. In the course of the above segment of talk, participants number the slots (lanes) on the film in order to identify their content by moving from the right to the left. In this process, they pause to look at the bands that appear in the lanes they are in, compare adjacent bands, and pursue questions suggested by their appearance. The moves and turns made by the gaze as it travels across the image are locally induced; local features of the display equally determine the departures from its surface, that is, the visits to the lab and to other contexts previously mentioned.

\section{Filling in the Test Tube}

In segments of image arithmetic, departures from the film are brief and devoted to aiding the calculations performed; in other segments, departures are extensive, repeated, and focussed on problems that may not have quick solutions. Because such problems are pervasive, visits to contexts other than the image itself are frequent. As suggested before, it is the frequent occurrence of features on the film that somehow deviate from what one desires and expects that gives rise to the pathology work of image dissection. Pathology work seeks to identify the cause of the trouble spots on the film. Each data spot on a display is a mark from which invisible threads lead to particular occurrences in experimental processes - threads that participants pursue in image dissection. 
The following example is drawn from the third of a series of image inspections that turned around the film on which the above image arithmetic was initially performed. Participants considered which events during various stages of an "S1-digestion" could possibly account for the bands encountered on their film. The film, as implied before, had not turned out ideal. Among other things, most of the bands of one marker were missing, which made it more difficult to identify the length of other bands. But the main trouble was that while the marker bands were missing, many more bands than expected had turned up in other lanes. Ideally, the bands on the autoradiograph would have corresponded approximately to the left side of Figure 6 . In practice, the autoradiograph looked more like the right side.

The troublesome bands were those that appeared between the uppermost marker band to the left and the bottom of the film. The discussion turned around which of these bands were the correct starts and what the other bands represented. For some bands to be plausibly identified as starts, all others have to be accounted for as something else. As they considered various spots on the film, participants envisaged more and more undesirable products to have possibly been part of the reaction mixtures poured into the gel-slots. In other words, they filled in the test tube in which a particular biochemical reaction, "S1 digestion," takes place shortly before the gel is poured.

Ideally, S1-digestion is a clean-up operation: it "clears" the reaction mixture from all but the desired genetic products before these products are made visible through an autoradiograph exposed on an electrophoresis gel. Strictly speaking, the S1-Nuclease does not eliminate the DNA and RNA strands it attacks, but only cuts them shorter. ${ }^{13}$ It hereby creates the possibility of fragments that are only partially digested (left too long) because there has not been enough S1. Also, the S1-Nuclease only attacks single strands. Double stranded fragments, such as "false starts" (i.e., longer or shorter than expected hybrids), double stranded probes, or DNA and RNA folded back upon itself are not digested by $\mathrm{S} 1$ and may remain in the mixture. Finally, S1-Nuclease can attack correct, double stranded hybrids through a hole in one strand, thereby creating bands that represent the desired start sides but appear to be artifacts because they are too short. Ideally, the mixture obtained after S1-digestion would have included no more than the products listed in Figure 7. After participants' image dissection, the test tube contained the additional products indicated in Figure 8.

Filling in the test tube is a process of envisaging the specimen behind the trouble spots on the image. Since most of these specimens are not visible to the eye, it is a process of glossing the invisible through the language of 


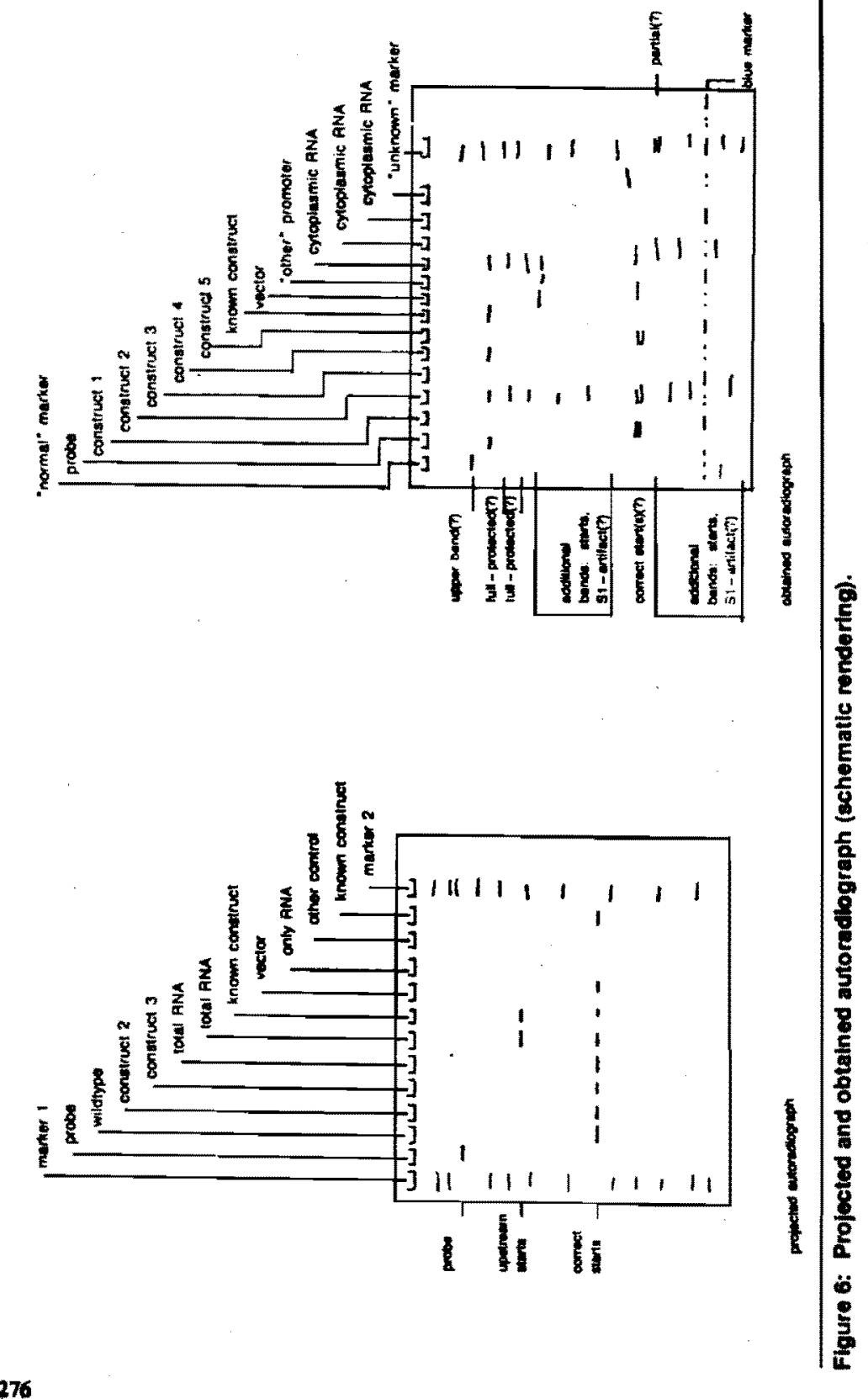




\section{- singlestranded probe \\ - DNARNA hybrid \\ - S1-Nuclease \\ 口 salt \\ $=$ carrier-DNA}

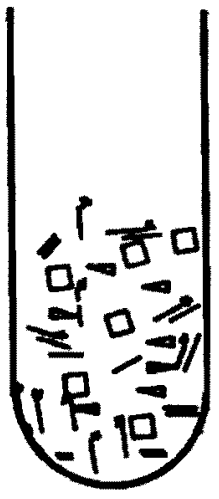

Figure 7. Projected mix of producte reaulting from \$1-dlgestion.

technical design exemplified above. Ostensibly, participants point to laboratory occurrences; however they grasp these occurrences in terms of visual formulations (see Figure 9).

Filling in the test tube, then, is a two-step process. It involves moving through the lab, behind the scenes, to stop at the backstage of experimental events. It is a process of simultaneously "appresenting" - to use Schutz's term - the context of embodied laboratory practices and the domain of the invisible to which these practices apply. Attempts to account for the visual features of an image may also refer exclusively to the lab, in which case the work achieved reconstructs the history of procedural manipulations. ${ }^{14}$ But problem spots on the film do not always give rise to account sequences. They nay also spur consideration of remedial actions, as when scientists regard the future of the display.

\section{Images by Design}

Interestingly, remedial considerations occur in image-attached conversations independently of whether the question of what the problem spots on the display represent has been solved or even been raised. The issue whether and 
double stranded probe

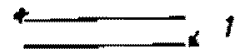

single stranded probe and hybridized

RNA with overlapping ends

(not digested by S1)

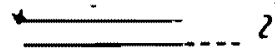

FNA lolded back upon itself

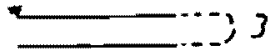

single stranded probe lolded back upon itseif

+..... 4

incomplete ("partial") digestion of single stranded probe

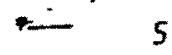

nicked DNA (6), overdigestion of S1 (7)

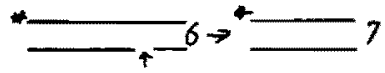

false start sides resulting in shorter (8) or longer hybrids (9)

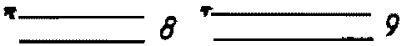

additional transcription products from other start sides resulting

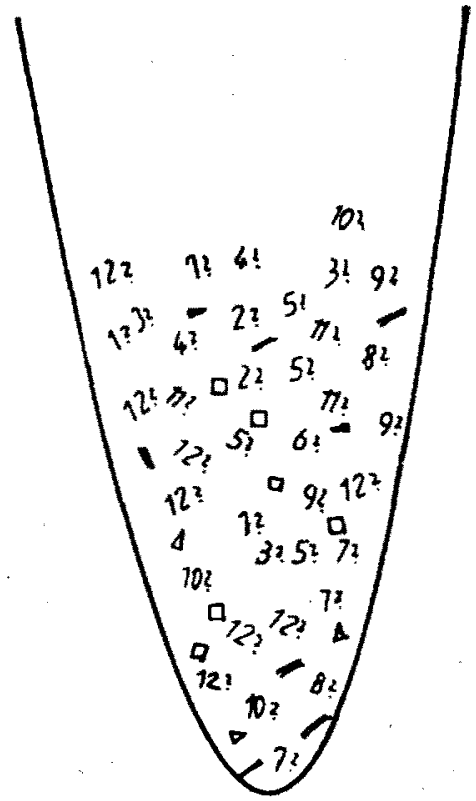
in shorter (10) or longer (11) hyorids

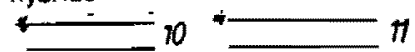

correct start sides resulting in hybrids of expected length

- $\mathbf{S 1 - N u c l e a s e}$

- salt

$=$ carrier - DNA

Figure 8. Reaction mix after three rounde of image inspection.

under what circumstances a display can be published appears to be always close to hand in such talk. It frequently marks the end of a round of talk; but it may also be brought up right in the beginning, and throughout the course of the conversation. Problem features of a data display can be remedied even 


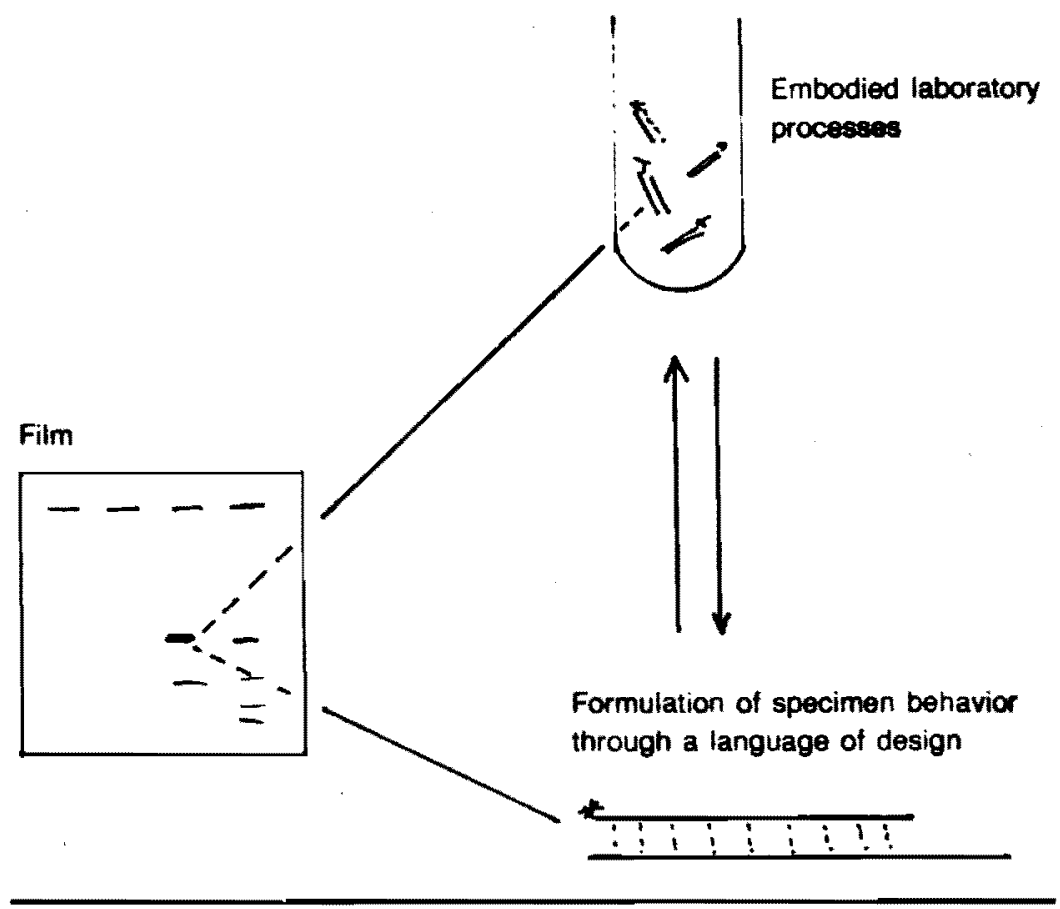

Figure 9. The two-step process of filling in the test tube.

if they cannot be readily explained. One way to conclude an unsatisfying attempt at explaining such features is to resort to such negative solutions - to ways of undoing the problem without solving it.

The following passage is drawn from the same conversation as the accounting procedure spelled out above. It includes an unusually complete list of possible autoradiograph manipulations that can help in turning a data display into an evidence exhibit, that is, in transforming the proto-data of the lab into publicly showable proofs: (1) Cutting off bands before photographing the image for publication; (2) taking less probe; (3) labeling some bands as "partials" in combination with cutting others off; (4) repeating the experiment; (5) exposing the $X$-ray film for a shorter period of time to prevent certain bands from appearing; (6) applying more S1-Nuclease in a repeat run to eliminate the specimen that created these bands, in combination with cutting off remaining troublesome bands; and (7) letting some bands "run out" of the gel by turning the electrophoresis apparatus off at an appropriate 
point in time. These are all ways to "disappear" the disturbing problem spots on the film.

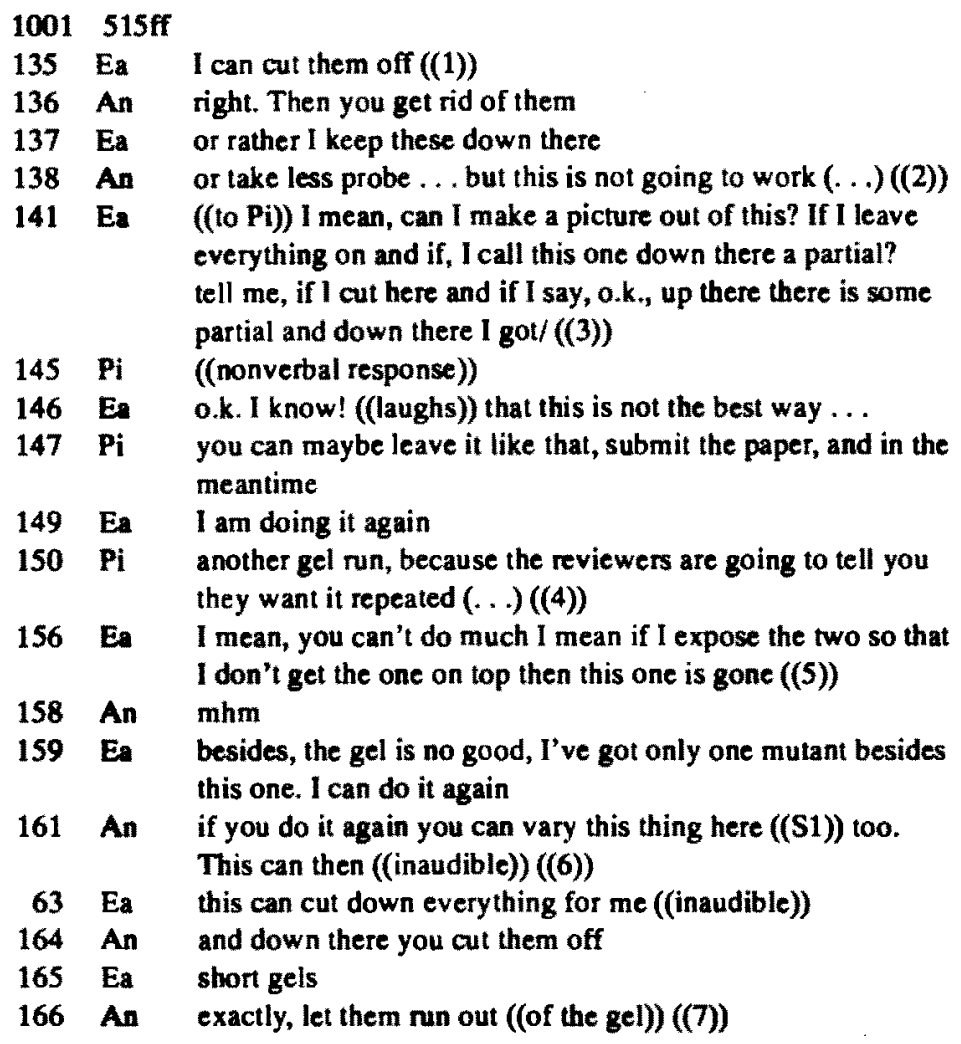

The resulting image is an image by design. It may be created through a clever combination of an experimental set up specifically designed to produce an image for publication, remedial actions applied to the image after it was experimentally obtained, and labeling strategies. It may take into account reference pictures published by other scientists that participants have on file, or imagined responses by reviewers to whom the image might be referred. Presumably, the image shapes-up and assumes its ideal form under the eyes of participants as they dryly envisage these options. The results are evidence advertisements that carry their message within themselves; they are "showable" rather than "tellable" arguments. Participants are never concerned with how one might verbally argue a particular knowledge claim in this context. 


\section{Conclusion}

Image surface calculations, reconstructions of events in the test tubes of the lab, and remedial actions designed to transform badly tumed-out pictures into showcases of data exemplify the type of work performed when technical images are inspected in the laboratory. Suffice it to add that many autoradiographs or other images are not just inspected once, but give rise to several image-related conversations, and many images are internally related by being predecessors or successors of others. In a series of related image inspections, some image conversations focus as a whole on certain kinds of work. For example, they may "zoom in" on the question of whether certain bands on the film represent the probe or a start, and they may do so by means of a dominant method (i.e., by envisaging the biochemical processes that may have given rise to these bands). In the case of the autoradiograph investigations from which the examples in this article are drawn, more than one image inspection focused more or less entirely on the above question; but one attacked the question by the predominant method of comparing the autoradiograph with altogether seven others that had been obtained in the labo-

ratory or were published in the literature of the field. For a brief period of time, the analysis was "locked into" the exploration of case precedents through whose medium the events in the test tubes that gave rise to the image were pursued. Thus, instead of looking more or less directly at the laboratory and glossing the invisible processes therein, participants looked first at other pictures and let themselves be guided to these processes by the appearance of the pictures. The example illustrates that there are variations on the procedures that participants combine in image dissection. In evaluating participants' performance in a particular round of talk, one must be aware of the fact that they might, at other times, produce a different performance. The examples we have given were taken from those rounds in a series of image inspections that contained a great variety of image-related work. In the cases investigated, these inspections occurred early on in the series. Participants' early investigations of the image offer a broader picture of image processing in the laboratory than image inspections that come late in the series. The question not pursued in this article is how interpretations of the image change as participants move through many rounds of inspection.

\section{Notes}

1. As an example for this perspective, consider Derrida's (1976) view that the idea of science was bom in a particular epoch of writing, or Peirce's (1955) attempt to reconstruct the 
logic of science in terms of a semiotic of written discourse. Other authors imply that certain forms of written discourse cannot be fully understood without an analysis of the practices from which they originate. Cicourel (1974) has repeatedly made this point in regard to questionnaire data in sociology.

2. For a somewhat different opinion, see Geertz (1973, chap. 1) and Latour and Woolgar (1979). Both argue that scientists are compulsory writers, through Geertz's claim refers only to social scientists.

3. For a different example of the use of images in natural science inquiry, see Lynch (1985b)

4. The present research includes the study of laboratories in four scientific fields: particle physics, molecular genetics, artificial intelligence, and transsexual research. All studies are in progress. For examples of other laboratory studies, see Knorr-Cetina (1981), Latour and Woolgar (1979), Lynch (1985a), Traweek (1988), and Zenzen and Restivo (1982). For papers reporting on the present research, see Amann and Knorr-Cetina (1989), and Knorr-Cetina (1988).

5. See "Filling in the test tube" discussed below. For a description of transcriptional control mechanisms and the methods of studying these, see Alberts et al. (1983).

6. The "start" refers to the initiation point of RNA produced in transcription, and indirectly to the length of the RNA as indicated by a particular location of RNAbands on an autoradiograph.

7. See Amann and Knor-Cetina (1988).

8. See Amann and Knorr-Cetina (1988).

9. Our impression is that this holds not only for image-attached conversations but for many other kinds of shop talk as well.

10. By conversation analysis standards (Sacks et al, 1974), the following data are fairly grossly transcribed. We have neglected overlaps and omitted indications of the length of pauses, and we have not transcribed explosive aspiration, "latching," or prolonged prior syllables. We believe, however, that the transcriptions are adequate for the level of analysis we attempt in this article, and we feel that they are easier to read for the audience to whom the article is addressed. The following transcribing conventions were uod:

I Interruption

() Single paren theses indicale the transcriber was not sure about the words contained within parentheses. Empty parentheses indicate talk inserted in or before passages relevant to the case presented.

(( )) Double parentheses indicate comments by the transcriber.

? Rising intonation Falling intonation, not necessarily end of sentence.

11. Scientists not present at an experiment (such as heads of laboratories) are considered to be prone to produce misjudgments. See Knorr-Cetina (1988).

12. In the present case, the situation is complicated by the fact that only the top band of the hpa II marker to which Ea refers is visible, and its length appears to be itself inferred from the position of the probe on the film and of the blue marker on the gel. Normally, the length of marker bands is determined by comparing the bands on the film with a published reference spectrum of bands for this marker.

13. It thereby prevents these materials from showing on the autoradiogaph, because the image only covers a certain range of length in DNA and RNA strands.

14. For a description of the machinery of conversation employed in reconstructing the history of procedural manipulations "behind" a technical effect, see Amann and Knorr-Cetina (1989). 


\section{References}

Alberts, B., D. Bray, J. Lewis, M. Raff, K. Roberts, and J. D. Wetson. 1983. Moleculer biology of the cell. New York: Garland.

Amann, K, and K. Knorr-Cetina. 1988. The fixation of visual evidence. Human Studies 2:135-169.

1989. Thinking through talk: An ethnographic study of a molecular biology laboratory. In Knowledge and sociery: Studies in the sociology of science past and present, edited by R. A. Jones, L. Hargens, and A. Pickering, 3-26. Greenwich, CT: JAI.

Cicourel, A. 1974. Interviewing and memory. In Pragmatic aspects of human communication, edited by C. Cherry, 51-82. Dordrecht, Holland: D. Reidel.

Derrida, J. 1976. Of grammatology. Bal timore: Johns Hopkins University Press.

Geertz, C. 1973. The interpretation of cultures. New York: Basic Press.

Knorr-Cetina, K. 1981. The manufacture of knowledge: An essay on the constructivist and contexrual nature of science. Oxford: Pergamon.

1988. Das Labor als Verdichtung von Gesellschaft. Zeitschrift für Soxiologie Vol. 17 (2):85-101.

Latour, B., and S. Woolgar. 1979. Laboratory life: The social construction of scientific facts. Beverly Hills, CA: Sage.

Lynch, M. 1985a. Art and artefact in laboratory science: A stucty of shop work and shop talk in a research laboratory. London: Routledge \& Kegan Paul.

1985b. Discipline and the material form of images: An analysis of scientific visibility. Social Studies of Science 15:37-66.

Peirce, C. S. 1955. Logic as semiotic. In Philosophical writings of Peirce, edited by J. Buchler. New York: Dover.

Sacks, H., E. Schegloff, and G. Jefferson. 1974. A simplest systematics for the organization of turn-laking in conversation. Language 50: 696-735.

Traweek, S. 1988. Beamtimes and lifetimes: The world of high energy physicists. Cambridge: Harvard University Press.

Zenzen, M., and S. Restivo. 1982. The mysterious morphology of immiscible liquids: A study of scientific practice. Social Science Information 21:447-73.

Karin Knorr-Cetina is Professor of Sociology at the University of Bielefeld, West Germany. She is author of The Manufacture of Knowledge (Oxford, 1981) and Co-Editor (with M. Mulkay) of Science Observed (London, 1983) and Advances in Social Theory and Methodology (London, 1981). She is currently finishing a book How Scientists Make Sense.

Klaws Amann is Lecturer of Qualitarive Methods in Sociology at the University of Bielefeld, West Germany. He just finished his dissertation on Natural Expertise and Artificial Intelligence. 